\title{
Smooth muscle antibodies and cryoglobulinemia are associated with advanced liver fibrosis in Brazilian hepatitis $C$ virus carriers
}

\author{
Authors \\ Luis Jesuino de Oliveira \\ Andrade $^{1}$ \\ Paulo Roberto Santana \\ de Melo $^{2}$ \\ Ajax Mercês Atta ${ }^{3}$ \\ Maria Luiza Brito de \\ Sousa $\mathrm{Atta}^{3}$ \\ Larissa Santana de Jesus ${ }^{4}$ \\ Gabriel Menezes de \\ Sousa $^{4}$ \\ Carolina Alves Costa \\ Silva ${ }^{5}$ \\ Raymundo Paraná ${ }^{6}$ \\ ${ }^{1} \mathrm{PhD}$; Faculdade de \\ Medicina da Universidade \\ Estadual de Santa Cruz, \\ Ilhéus, Bahia, Brazil \\ ${ }^{2} \mathrm{PhD}$; Department of \\ Biological Sciences; \\ Universidade Estadual de \\ Santa Cruz; Bahia; Brazil \\ ${ }^{3} \mathrm{PhD}$; Department of \\ Toxicological and Clinical \\ Analysis; Faculdade de \\ Farmácia, Universidade \\ Federal da Bahia; \\ UFBA - Brazil \\ ${ }^{4}$ Post-Graduate; \\ Department of \\ Toxicological and Clinical \\ Analysis, Faculdade de \\ Farmácia, UFBA, Brazil \\ ${ }^{5}$ Medicine Student; \\ Gastro-Hepatology Service, \\ Complexo Hospitalar \\ Professor Edgard Santos, \\ Faculdade de Medicina da \\ Bahia, UFBA,Brazil \\ ${ }^{6} \mathrm{PhD}$; Gastro-Hepatology \\ Service, Complexo \\ Hospitalar Professor Edgard \\ Santos, Faculdade de \\ Medicina da Bahia, UFBA, \\ Brazil
}

Submitted on: 03/25/2010 Approved on: 05/21/2010

Correspondence to: Luis Jesuino de Oliveira Andrade

Rua Nações Unidas, 511 - Centro 45600-673 Itabuna, Bahia, Brazil Phone: +557388122755 luis_jesuino@yahoo.com.br

We declare no conflict of interest.

\begin{abstract}
Cryoglobulinemia and non-organ-specific-autoantibody are biomarkers of autoimmunity of the chronic infection caused by hepatitis $\mathrm{C}$ virus (HCV). In this work, we report the association between the presence of smooth muscle antibodies (SMA) and cryoglobulinemia and chronic liver disease in HCV carriers. Sixty-five untreated HCV patients, 38 women and 27 men were included in this study. Cryoglobulinemia was tested by cryoprecipitation, SMA by indirect fluorescent antibody test, and liver fibrosis and hepatocellular inflammation activity was investigated by histology of liver biopsy using the METAVIR score. The prevalence of SMA in the patients was $33.8 \%$ and cryoglobulinemia was demonstrated in $36.9 \%$ patients. Cryoglobulinemia and SMA seropositivity was associated with advanced fibrosis $(\mathrm{p}<0.05)$. The presence of SMA and cryoglobulinemia was not associated with hepatocellular inflammation activity, age, carrier gender or HCV genotype. We concluded that liver biopsy should be recommended for HCV carriers that are seropositive for SMA or cryoglobulinemia.
\end{abstract}

Keywords: hepatitis C; muscle; smooth; antibody specificity; cryoglobulins; liver diseases.

[Braz J Infect Dis 2011;15(1):66-68] CElsevier Editora Ltda.

\section{INTRODUCTION}

Patients chronically infected by hepatitis $\mathrm{C}$ virus (HCV) present various immune-mediated phenomena mainly due to B lymphocyte dysfunction as mixed cryoglobulinemia and non-organ-specific autoantibodies (NOSAs) production. ${ }^{1,2}$ Although cryoglobulinemia has been associated with vasculitis of small and medium-size vessels and glomerulonephritis, the involvement of NOSAs in the immunopathology of hepatitis $\mathrm{C}$ is not completely understood. However, the presence of smooth muscle antibodies (SMA) has been reported to be higher in cryoglobulinemic HCV carriers, whereas cryoglobulinemia has been associated with advanced liver fibrosis and poor response to antiviral therapy with interferon- $\alpha .^{3,4}$

In this study we report the association between SMA, cryoglobulinemia and liver fibrosis in untreated Brazilian HCV carriers.

\section{PATIENTS AND METHODS}

\section{Patients}

Sixty-five consecutive untreated HCV carriers ( 38 women and 27 men), with a mean age of $51.3 \pm 13.9$ years (ranging from 20 to 67 years), from two hepatitis reference centers in Salvador (Bahia, Brazil) were included in this study. They tested positive for serum anti-HCV antibodies by enzyme-linked immunosorbent assay with structural and non-structural HCV antigens (AXSYM System; Abbott Laboratories, Chicago, IL, USA) and for HCV-RNA by polymerase chain reaction (PCR) (Amplicor ${ }^{\mathrm{TM}} \mathrm{HCV}$ Detection KIT V2.0; Roche Molecular Systems Inc., Somerville, NJ, USA). The median ALT level was $56 \mathrm{U} / \mathrm{L}$ and $65.5 \mathrm{U} / \mathrm{L}$ for the women and men, respectively. Forty-five carriers were infected with $\mathrm{HCV}$ genotype 1 , and 20 with the genotype 3 as determined by the INNOLIPA test (Innogenetics, NV, Gent, Belgium).

An informed consent was signed by all patients to participate in the study, which was approved by the Ethical Committee of Universidade Estadual de Santa Cruz, Bahia, Brazil.

\section{Liver histopathology}

Histology of liver biopsy specimens was performed by a single experienced liver pathologist in samples stained by hematoxylin-eosin, Picrosirius red, and Perls stains. To avoid bias, the clinical background of the donors of liver biopsies was not known by this professional before the exams. 
The histological abnormalities in liver biopsy were classified in accordance with the METAVIR system: ${ }^{5}$ F0, no fibrosis; F1, portal fibrosis without septa; F2, few septa; F3, numerous septa without cirrhosis; and F4, cirrhosis. Necroinflammatory activity was graded on a scale of A0-A3, corresponding A0 to no histological activity; A1, mild activity; A2, moderate activity; and A3, severe activity.

\section{Cryoglobulinemia and SMA}

Cryoglobulin was investigated using both tube and gel cryoprecipitation. ${ }^{6}$ Smooth muscle IgG antibodies were tested by indirect fluorescent antibody test using antigenic substrates of rodent rat liver-kidney-stomach sections and sera diluted at 1:40.

\section{Statistical analysis}

The association between two or more categorical groups was analyzed by the Exact Fisher test or the chi-square test, using the statistical software SPSS ${ }^{\mathrm{TM}}$ version 16.0 for Windows. A p-value $<0.05$ was considered significant.

\section{RESULTS}

Varied degrees of fibrosis were observed in the HCV carriers. Forty-four out of $65(67.7 \%)$ carriers had mild to moderate fibrosis (F1-F2), 16 out of 65 (32.3\%) had advanced fibrosis (F3) and five (7.7\%) had cirrhosis (F4). No inflammatory activity (A0) was observed in $13 \mathrm{HCV}$ carrier. Seven of them had a mild inflammatory activity (A1), 30 presented a moderate activity (A2) and in only two individuals it was severe.

Smooth muscle antibodies were detected in 22 out of 65 (33.8\%) HCV carriers and cryoglobulinemia was found in 24 out of 65 (36.9\%) patients. Advanced liver fibrosis (F3F4) was associated with the presence of smooth muscle antibodies (Fisher exact test, $\mathrm{p}=0.048$ ), and progression to advanced liver fibrosis with the presence of cryoglobulinemia (chi-square test, $\mathrm{p}=0.031$ ) (Figure 1 ).

Neither smooth muscle antibodies nor cryoglobulinemia was associated with hepatocellular inflammatory activity, age, carrier gender or any HCV genotype.

\section{DISCUSSION}

In this study, we demonstrated the association of SMA and cryoglobulinemia with liver fibrosis in Brazilian HCV carriers. Smooth muscle antibodies are immunoglobulins that react with filaments of the cytoskeleton, which are represented by microfilaments (actin and vinculin), intermediate filaments (vimentin and desmin) and microtubules (tubulin). Although these autoantibodies are more commonly detected in autoimmune hepatitis, mainly anti-actin antibodies, they may also be found in low titers and varied prevalence in normal individuals and in patients presenting different infections, cancer and systemic autoimmune diseases. Smooth muscle antibodies are frequently detected in sera of $\mathrm{HCV}$ carriers as previously reported by several authors, with a prevalence varying from $10 \%$ to $55 \% .^{6}$ The prevalence of SMA found in HCV carriers in this work was in accordance with the findings of a previous Brazilian study and also in accordance with the reports of hepatitis $\mathrm{C}$ studies performed in different countries. ${ }^{7-9}$

Hepatic fibrosis is caused by a complex immune mechanism, influenced by genetic and environmental factors, which involves cytokines as TGF-beta, angiotensin II and leptin. Inflammatory cells as polymorphonuclear cells and lymphocytes, and also activated hepatic stellate cells, portal fibroblasts and myofibroblasts participate in this process that culminates with excessive secretion of matrix proteins including collagen. ${ }^{10}$ Our results found the association between SMA seropositivity and advanced fibrosis (F3-F4) that may be an additional evidence of the involvement of the Th2 immune response in the active fibrogenesis as previously revised. ${ }^{11}$ The association between SMA and advanced fibrosis was also reported in a recent study performed with French HCV carriers, which also demonstrated an association between the presence of SMA and hepatocellular necrosis and inflammation, ${ }^{12}$ a finding that was not verified in our work. Such a divergence related to hepatic

Figure 1: Distribuition of liver fibrosis score in HCV carries seropositive and seronegative for cryoglobulinaemia.

Cryoglobulinaemia was associated with liver fibrosis progression (chi-square test, $\mathrm{p}<0.05$ ).

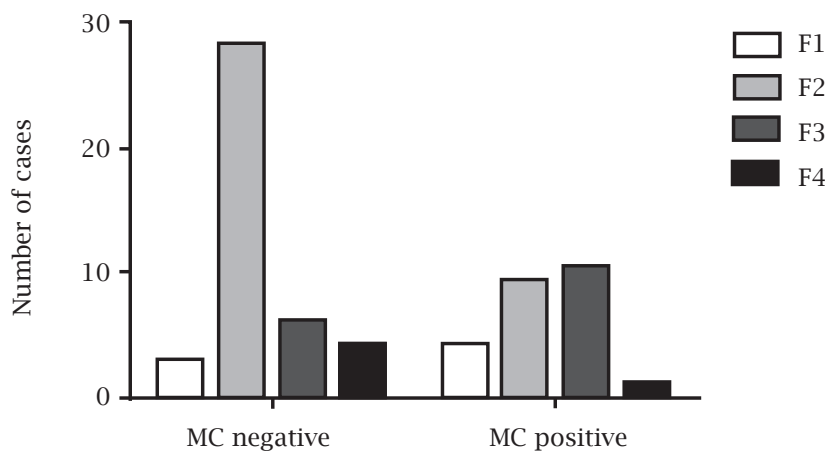


necroinflammation may be caused by ethnic difference in the populations studied or by difference in the genetic background of the HCV carriers investigated. This possibility may be supported by the association of smooth muscle antibodies with HLA A1-B8-DR3 haplotype. ${ }^{13}$ Nevertheless, the involvement of molecular homology between HCV proteins and human self-antigens in the production of SMA has been documented. ${ }^{14}$

Cryoglobulinemia is the main extra-hepatic manifestation of chronic hepatitis $\mathrm{C}$, which is caused by a dysfunction of B-lymphocyte and is immunochemically characterized by a cryoprecipitable immune complex formed by rheumatoid factor and IgG antibodies linked to $\mathrm{HCV}$ core antigen. Its prevalence varies from $29 \%$ to $54 \%$ in HCV carriers, and has been clinically associated with leg ulcers, vasculitis, palpable purple skin lesions and glomerulonephritis. ${ }^{15}$ The statistically significant association between CM positivity and advanced fibrosis in our study was in agreement with results of a Greek study that reported an association between cryoglobulinemia and staging score of chronic HCV infection. ${ }^{4}$ Nevertheless, this association is still controversial and need more studies to elucidate and establish the link between these two immunopathological aspects of chronic hepatitis C.

We concluded that liver histology should be performed in untreated HCV carriers that are seropositive for either smooth muscle antibodies or cryoglobulinemia.

\section{REFERENCES}

1. Strassburg CP, Vogel A, Manns MP. Autoimmunity and hepatitis C. Autoimmun Rev. 2003; 2:322-31.

2. Dustin LB, Rice CM. Flying under the radar: the immunobiology of hepatitis C. Annu Rev Immunol. 2007; 25:71-99.

3. Lunel F, Musset L. Hepatitis C virus infection and cryoglobulinemia. J Hepatol. 1998; 29:848-55.

4. Siagris D, Christofidou M, Tsamandas A, Lekkou A, Thomopoulos K, Labropoulou-Karatza C. Cryoglobulinemia and progression of fibrosis in chronic HCV infection: cause or effect? J Infect. 2004; 49:236-41.

5. Bedossa P, Poynard T. The METAVIR cooperative study group. An algorithm for the grading of activity in chronic hepatitis C. Hepatology 1996; 24:189-93.

6. Okazaki T, Nagai T, Kanno T. Gel diffusion procedure for the detection of cryoglobulins in serum. Clin Chem. 1998; 44:1558-9.

7. Ramos-Casals M, Font J. Extrahepatic manifestations in patients with chronic hepatitis $\mathrm{C}$ virus infection. Curr Opin Rheumatol. 2005; 17:447-55.

8. Atta AM, Oliveira IS, Sousa GM, Paraná R, Sousa Atta ML. Serum cytokine profile in hepatitis $\mathrm{C}$ virus carriers presenting cryoglobulinaemia and nonorgan-specific autoantibodies. Microb Pathog. 2010; 48:53-6.

9. Clifford BD, Donahue D, Smith L et al. High prevalence of serological markers of autoimmunity in patients with chronic hepatitis C. Hepatology 1995; 21:613-9.

10. Bataller R, Brenner DA. Liver fibrosis. J Clin Invest. 2005; 115:209-18.

11. Bhogal RK, Bona CA. B cells: no longer bystanders in liver fibrosis. J Clin Invest. 2005; 115:2962-5.

12. Chrétien P, Chousterman M, Abd Alsamad I et al. Non-organspecific autoantibodies in chronic hepatitis $\mathrm{C}$ patients: association with histological activity and fibrosis. J Autoimmun. 2009; 32:201-5.

13. Bantel H, Kneser J, Manns MP. Autoantibodies and organspecific autoimmunity. In: Autoantibodies and Autoimmunity: Molecular Mechanisms in Health and Disease. Pollard KM (Ed) 2006 WILEY-VCH Verlag GmbH \& Co. KGaA, Weinheim.

14. Gregorio GV, Choudhuri K, Ma Y et al. Mimicry between the hepatitis $\mathrm{C}$ virus polyprotein and antigenic targets of nuclear and smooth muscle antibodies in chronic hepatitis $\mathrm{C}$ virus infection. Clin Exp Immunol. 2003; 133:404-13.

15. Sansonno D, Dammacco F. Hepatitis C virus, cryoglobulinaemia, and vasculitis: immune complex relations. Lancet Infect Dis. 2005; 5:227-36. 